\title{
Photon shell game in three-resonator circuit quantum electrodynamics
}

\author{
Matteo Mariantoni ${ }^{1,2 \star}$, H. Wang ${ }^{1}$, Radoslaw C. Bialczak ${ }^{1}$, M. Lenander ${ }^{1}$, Erik Lucero ${ }^{1}$, M. Neeley ${ }^{1}$, \\ A. D. O'Connell', D. Sank ${ }^{1}$, M. Weides ${ }^{1}$, J. Wenner ${ }^{1}$, T. Yamamoto ${ }^{1,3}$, Y. Yin ${ }^{1}$, J. Zhao', \\ John M. Martinis ${ }^{1}$ and A. N. Cleland ${ }^{1 \star}$
}

The generation and control of quantum states of light constitute fundamental tasks in cavity quantum electrodynamics ${ }^{1-10}$ (QED). The superconducting realization of cavity QED, circuit QED (refs 11-14), enables on-chip microwave photonics, where superconducting qubits ${ }^{15-18}$ control and measure individual photon states ${ }^{19-26}$. A long-standing issue in cavity QED is the coherent transfer of photons between two or more resonators. Here, we use circuit QED to implement a three-resonator architecture on a single chip, where the resonators are interconnected by two superconducting phase qubits. We use this circuit to shuffle one- and two-photon Fock states between the three resonators, and demonstrate qubit-mediated vacuum Rabi swaps between two resonators. By shuffling superposition states we are also able to demonstrate the high-fidelity phase coherence of the transfer. Our results illustrate the potential for using multi-resonator circuits as photon quantum registers and for creating multipartite entanglement between delocalized bosonic modes ${ }^{27}$.

The combination of high-finesse electromagnetic cavities with atoms or qubits enables fundamental studies of the interaction between light and matter. The cavity provides a protected environment for storing and tailoring individual photonic excitations ${ }^{1-3,14}$. Both stationary ${ }^{6,21,23}$ and propagating ${ }^{7,9}$ non-classical fields can be synthesized using such systems, enabling quantum memory and quantum messaging ${ }^{8}$. A critical challenge however is the extension from single to more versatile multi-cavity architectures ${ }^{27,28}$, allowing manipulation of spatially separated bosonic modes. Although the entanglement of different modes of a single cavity ${ }^{4}$ and of free-space modes ${ }^{10}$ has been shown in atomic systems, and a coupled low- and high-quality-factor resonator studied in circuit QED (refs 25,26), coherent dynamics between two or more high-quality-factor cavities has yet to be demonstrated. Here we describe a triple-resonator system, where three high-quality-factor microwave resonators are coupled to two superconducting phase qubits (see Fig. 1). The qubits serve as quantum transducers ${ }^{29}$ that create and transfer photonic states between the resonators. Both Fock states and linear superpositions of Fock states are transferred, thus demonstrating a fully phase coherent process. The quantum transduction is carried out by means of purely resonant qubitresonator interactions, rather than dispersive coupling ${ }^{27}$, enabling rapid transfers between resonators with significantly different frequencies. As an important example, we demonstrate single-photon Rabi swaps between two resonators detuned by $\simeq 12,000$ resonator linewidths.
Figure 1a,b shows the main experimental elements, which comprise three coplanar waveguide resonators, $R_{a}, R_{b}$ and $R_{c}$, two phase qubits, $\mathrm{Q}_{1}$ and $\mathrm{Q}_{2}$, and two superconducting quantum interference devices used for qubit state readout. Each qubit is coupled to a control line that is used to adjust the qubit operating frequency $f_{1,2}$ and couple microwave pulses for controlling and measuring the qubit state. During operation, the device is attached to the mixing chamber of a dilution refrigerator at $\simeq 25 \mathrm{mK}$.

The circuit layout (see Fig. 1b) can be decomposed into two unit cells, $\mathrm{R}_{\mathrm{a}}-\mathrm{Q}_{1}-\mathrm{R}_{\mathrm{b}}$ (green area) and $\mathrm{R}_{\mathrm{b}}-\mathrm{Q}_{2}-\mathrm{R}_{\mathrm{c}}$ (blue area). The shared resonator $R_{b}$ connects the two cells and protects the two qubits from unwanted crosstalk. The resonator frequencies $f_{\mathrm{a}}, f_{\mathrm{b}}$ and $f_{\mathrm{c}}$ are measured with qubit spectroscopy (not shown). The vacuum Rabi couplings between each qubit and its corresponding resonators, $g_{1 \mathrm{a}}$ and $g_{1 \mathrm{~b}}$ for $\mathrm{Q}_{1}$ and $g_{2 \mathrm{~b}}$ and $g_{2 \mathrm{c}}$ for $\mathrm{Q}_{2}$, are determined by their respective coupling capacitors (see Fig. 1b). The coupling strengths are measured using two-dimensional swap spectroscopy (see Fig. 1c-e). We note that the swap spectroscopy provides an excellent tool for revealing the presence of spurious two-level systems as well as frequencies with short qubit relaxation times. In all of the experiments, the qubits are initialized in the ground state $|\mathrm{g}\rangle$ and are typically tuned to the idle point, where the qubit $\mathrm{Q}_{1}\left(\mathrm{Q}_{2}\right)$ $|\mathrm{g}\rangle \leftrightarrow \mid$ e $\rangle$ transition frequency $f_{1}\left(f_{2}\right)$ is set in-between, and well away from, the resonator transition frequencies $f_{\mathrm{a}}$ and $f_{\mathrm{b}}\left(f_{\mathrm{b}}\right.$ and $\left.f_{\mathrm{c}}\right)$.

When $Q_{1}\left(Q_{2}\right)$ is at the idle point, the qubit-resonator detuning is sufficiently large that the qubit-resonator interactions are effectively switched off. A particular qubit-resonator $\mathrm{Q}_{p}-\mathrm{R}_{q}$ ( $p=$ $1,2$ and $q=\mathrm{a}, \mathrm{b}, \mathrm{c})$ interaction is switched on by shifting the qubit transition frequency $f_{p}$ to equal the resonator frequency $f_{q}$, thus setting the detuning to zero and enabling quantum energy transfers. The time-dependent control of the qubit transition frequency consequently enables highly complex quantum control of the resonators ${ }^{23}$.

Figure $2 \mathrm{a}$ shows a diagram of the pulse sequence used to implement the single-photon equivalent of the 'shell game', in which a pea is hidden under one of three shells and the contestant must guess where the pea is after the shells have been shuffled. The three resonators play the role of the shells and a single-photon Fock state $|1\rangle$ that of the pea. The system is initialized in the ground state, with the qubits at their idle points, so that all interactions are effectively switched off. Qubit $Q_{1}$ is used to pump a single photon into resonator $\mathrm{R}_{\mathrm{a}}$ (see Fig. $2 \mathrm{a}(\mathrm{I})-(\mathrm{III})$ ). The photon state can then be transferred to either of the other two resonators, using the qubits in a similar fashion to mediate the single-excitation transfer. A

${ }^{1}$ Department of Physics, University of California, Santa Barbara, California 93106-9530, USA, ${ }^{2}$ California NanoSystems Institute, University of California, Santa Barbara, California 93106-9530, USA, ${ }^{3}$ Green Innovation Research Laboratories, NEC Corporation, Tsukuba, Ibaraki 305-8501, Japan.

*e-mail:matmar@physics.ucsb.edu; anc@physics.ucsb.edu. 


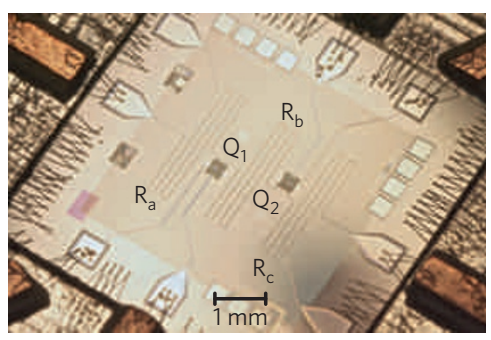

c
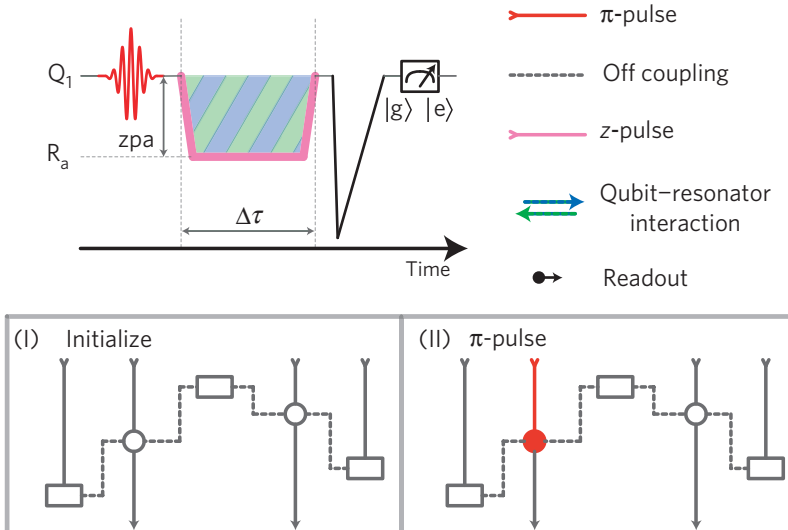

(III) z-pulse and interaction
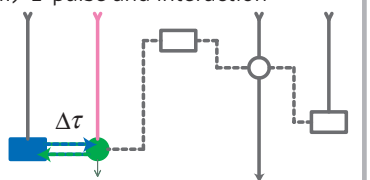

(IV

(IV) Readout

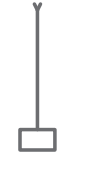

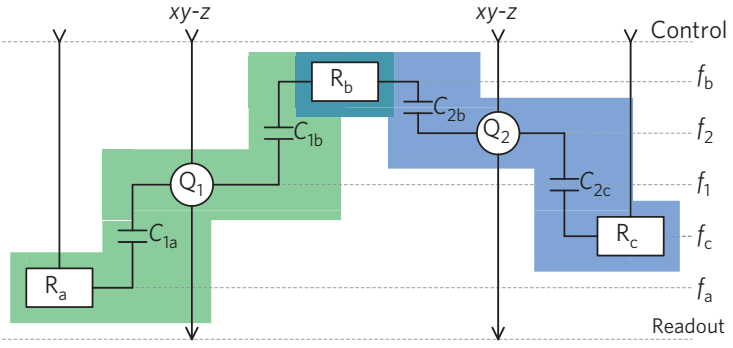

d

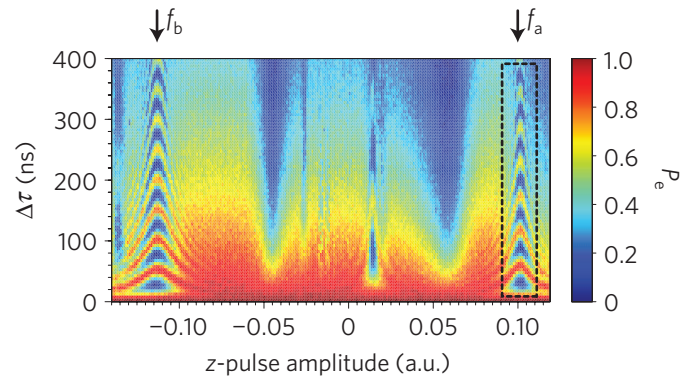

e

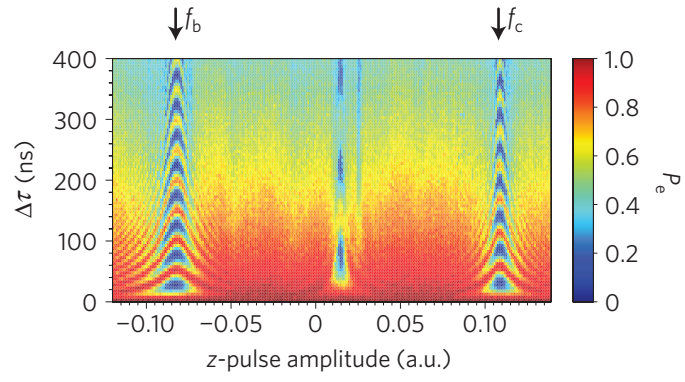

Figure 1 | Experimental architecture and two-dimensional swap spectroscopy. a, Photograph of a sample mounted on an aluminium holder, showing three coplanar waveguide resonators $\left(R_{a}, R_{b}\right.$ and $R_{c}$, with meander design) capacitively coupled to two superconducting phase qubits $\left(Q_{1}\right.$ and $\left.Q_{2}\right)$. $\mathbf{b}, B_{1}$ lock diagram showing the main elements, which comprise two circuit unit cells $R_{a}-Q_{1}-R_{b}$ (light green area) and $R_{b}-Q_{2}-R_{c}$ (dark blue area). The horizontal placement represents the spatial layout of the sample, whereas the vertical distribution corresponds to the frequencies of the elements. The qubit-resonator coupling capacitors are designed to be $C_{1 a}=C_{1 b}=C_{2 b}=C_{2 c}=1.9 \mathrm{fF} . f_{a} \simeq 6.29 \mathrm{GHz}, f_{b} \simeq 6.82 \mathrm{GHz}$ and $f_{c} \simeq 6.34 \mathrm{GHz}$ are the measured resonator frequencies, and $f_{1}$ and $f_{2}$ the tunable qubit transition frequencies. Control and readout wiring is also shown. c, Upper panel, pulse sequence for swap spectroscopy, with data shown in $\mathbf{d}$ and $\mathbf{e}$. The $\mathrm{Q}_{1}$ line shows a Gaussian microwave $\pi$-pulse (red) and a qubit tuning pulse ( $z$-pulse; hashed magenta) with variable $z$-pulse amplitude (zpa) and duration $\Delta \tau$, followed by a triangular measurement pulse (black). Lower panel, diagrammatic representation of the pulse sequence. (I), The entire system is initialized in the ground state. (II), $\mathrm{Q}_{1}$ is excited by a $\pi$-pulse (red), then, (III), brought into resonance with for example $R_{a}$ (dashed black rectangle in $\mathbf{d}$ ) by means of a z-pulse (magenta) and allowed to interact with the resonator (or electromagnetic environment) for a time $\Delta \tau$. (IV), A measurement pulse projects the qubit onto its ground state $|g\rangle$ or excited state $|\mathrm{e}\rangle$. d, Two-dimensional swap spectroscopy for $\mathrm{Q}_{1}$. The probability $P_{\mathrm{e}}$ to find the qubit in $|\mathrm{e}\rangle$ is plotted versus $z$-pulse amplitude and resonator measurement time $\Delta \tau$. The typical chevron pattern generated by a qubit-resonator swap (arrows) is evident for both the $Q_{1}-R_{a}$ (dashed black box) and $Q_{1}-R_{b}$ interactions. Near the centre of the plot a qubit interaction with a spurious two-level system is seen, surrounded by two regions with short qubit relaxation time. $\mathbf{e}$, The same as in $\mathbf{d}$ but for $\mathrm{Q}_{2}$. From these measurements, we find the coupling strengths $g_{1 \mathrm{a}} \simeq 17.58 \mp 0.01 \mathrm{MHz}, g_{1 b} \simeq 20.65 \mp 0.02 \mathrm{MHz}, g_{2 b} \simeq 20.43 \mp 0.01 \mathrm{MHz}$ and $g_{2 \mathrm{c}} \simeq 17.96 \mp 0.01 \mathrm{MHz}$.

transfer from $R_{a}$ to $R_{b}$ is shown in steps (IV)-(VI), and a second transfer from $\mathrm{R}_{\mathrm{b}}$ to $\mathrm{R}_{\mathrm{c}}$ carried out using $\mathrm{Q}_{2}$ in steps (VII)-(IX). The final location of the photon can be determined by employing the qubits as photon detectors, through the vacuum Rabi oscillations that occur when a qubit is brought in resonance with a resonator storing a photon (steps $(\mathrm{X})-(\mathrm{XI}))$.

In the data shown in Fig. $2 b(\mathrm{i})-(\mathrm{v})$, which represents different versions of the game, a photon was stored in one of the three resonators, shuffled between the resonators, and all three resonators then measured. In the shell game of Fig. $2 \mathrm{~b}(\mathrm{i})$, no photon was placed in any resonator, whereas for example in game (iv), a single photon was transferred from $R_{a}$ to $R_{c}$ through $R_{b}$, and then detected using qubit $\mathrm{Q}_{2}$; measurements of the other resonators $\mathrm{R}_{\mathrm{a}}$ and $\mathrm{R}_{\mathrm{b}}$ show no oscillations, that is, no photonic excitation.

We also explored a variant of the shell game, transferring a two-photon Fock state $|2\rangle$ from $R_{a}$ to $R_{b}$ to $R_{c}$. The twophoton Fock state (see ref. 21) is first generated in $R_{a}$, as shown by the measurements in Fig. 3a(i),(ii). Figure 3a also shows the measurements after this state is transferred from $R_{a}$ to $R_{b}$ and then to $R_{c}$. Each transfer takes two steps, starting with for example the state $\left|Q_{1} R_{a} R_{b}\right\rangle=|g 20\rangle$. The qubit is brought into resonance with $R_{a}$, and one photon is Rabi-swapped to the qubit, at a rate $\sqrt{2}$ faster than the usual one-photon rate ${ }^{21}$, leaving the system in the state $|e 10\rangle$. The qubit is then tuned into resonance with resonator $R_{b}$ for a one-photon Rabi swap, resulting in the state $|g 11\rangle$ (see Fig. 3a(iii)). The qubit is subsequently placed back in resonance with $R_{a}$ for a one-photon swap, yielding $|\mathrm{e} 01\rangle$, and brought into resonance with $R_{b}$ to transfer the second photon, ending with the state $|g 02\rangle$ (see Fig. 3a(iv)). To finally transfer the photons to resonator $R_{c}$, the process is repeated using qubit $\mathrm{Q}_{2}$, which completes the full transfer of Fock state $|2\rangle$ (see Fig. $3 \mathrm{a}(\mathrm{v})$ ). This process resembles the well-known game 'The towers of Hanoi', where a set of discs with different diameters has to be moved between three posts (the three resonators) while maintaining the larger discs (Fock 


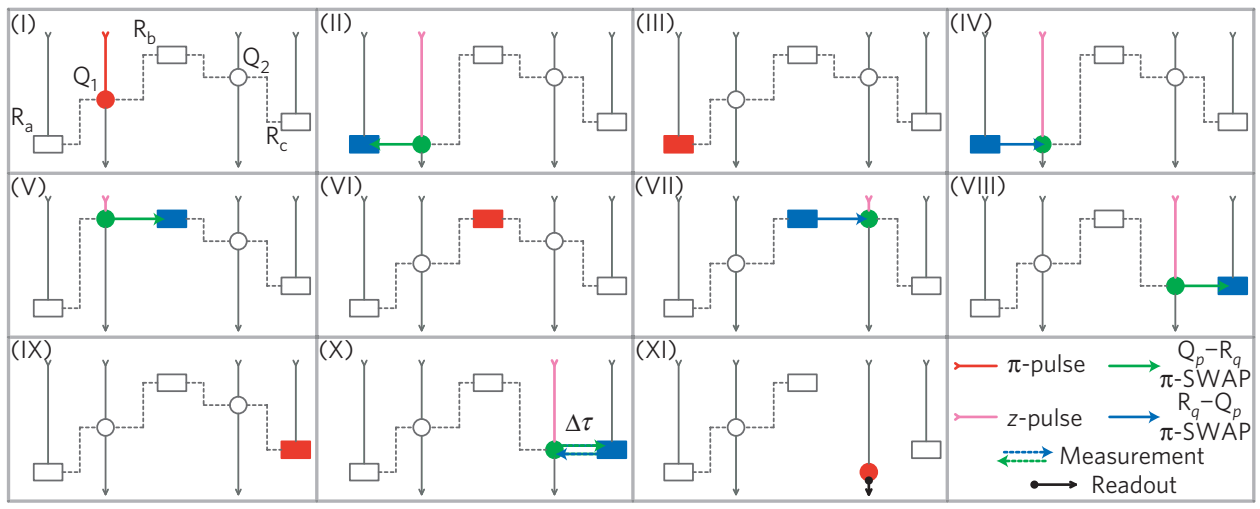

b
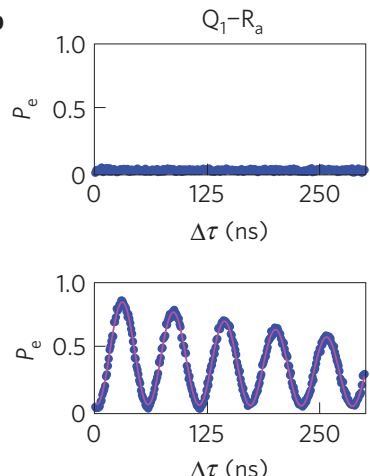

$\Delta \tau$ (ns)
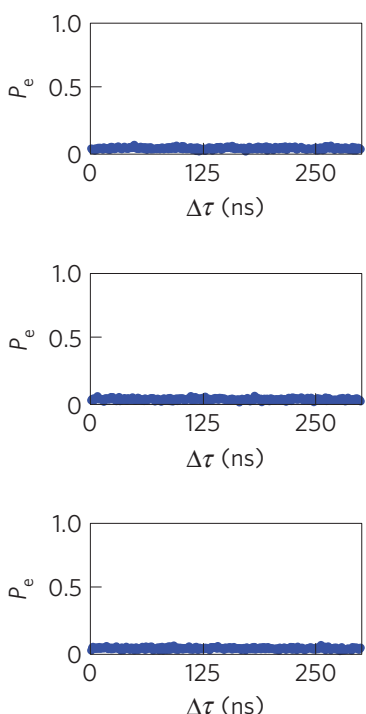
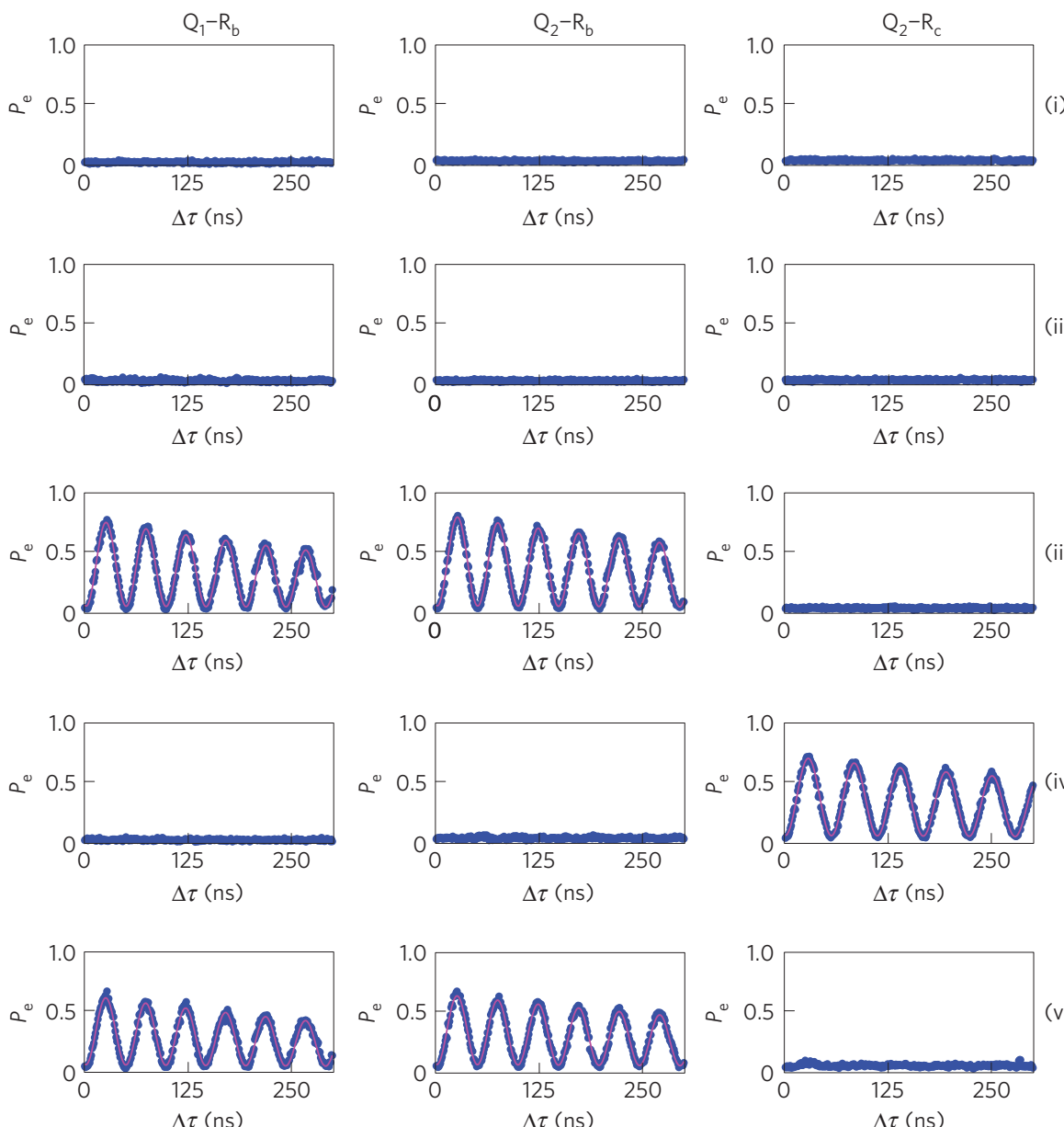

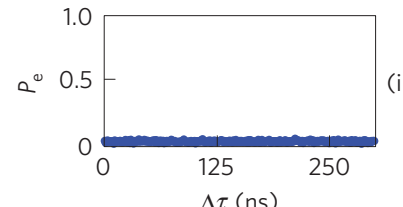

(ii)

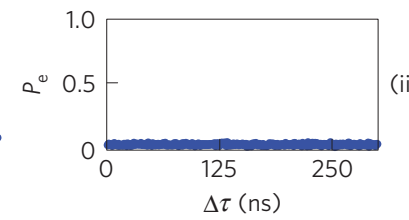

(iii)

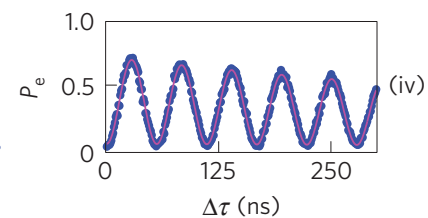

(i)
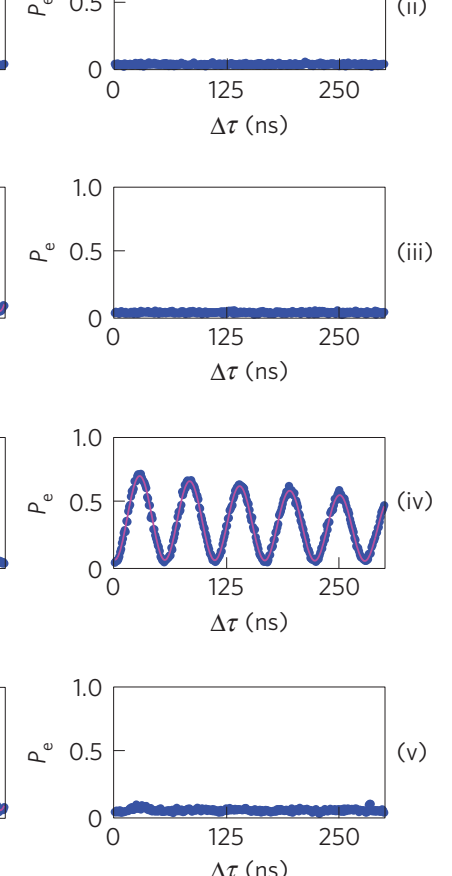

Figure 2 | Photon shell game. a, Block diagram of the sequence used to coherently transfer a single-photon Fock state $|1\rangle$ from $R_{a}$ to $R_{c}$ through $R_{b}$. After initializing the system in the ground state, (I), $Q_{1}$ is excited by a $\pi$-pulse and, (II), z-pulsed into resonance with $R_{a}$ for a full Rabi swap (Rabi $\pi$-swap) at the

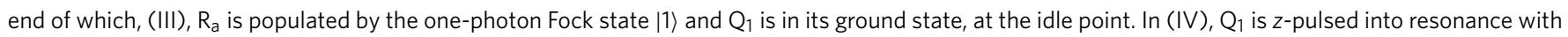
$\mathrm{R}_{\mathrm{a}}$ for a Rabi $\pi$-swap and then, $(\mathrm{V})$, z-pulsed into resonance with $\mathrm{R}_{\mathrm{b}}$ for another Rabi $\pi$-swap at the end of which, (VI), $\mathrm{R}_{\mathrm{b}}$ is populated by the one-photon Fock state and both $\mathrm{Q}_{1}$ and $\mathrm{Q}_{2}$ are in the ground state at the idle point. (VII), $\mathrm{Q}_{2}$ is z-pulsed into resonance with $\mathrm{R}_{\mathrm{b}}$ for a Rabi $\pi$-swap and, (VIII), z-pulsed into resonance with $R_{c}$ for another Rabi $\pi$-swap at the end of which, (IX), $R_{c}$ is in the one-photon Fock state and $Q_{2}$ in the ground state at the idle point. Measurement and qubit state readout are carried out in $(X)$ and $(X I)$, respectively, where the presence of the Fock state in $R_{c}$ is detected by its interaction with $\mathrm{Q}_{2}$. b. Measurement outcomes for different photon shell games. Each plot shows the probability $P_{\mathrm{e}}$ to measure a qubit in the excited state $|\mathrm{e}\rangle$ as a function of the qubit-resonator measurement time $\Delta \tau$. The blue circles are data; the magenta lines are a least-squares fit to an exponentially damped

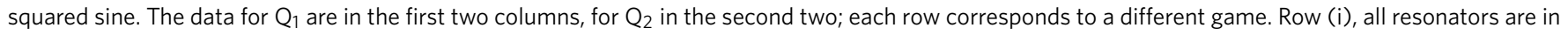
the vacuum state (with fidelity $\mathcal{F}>0.99$; we define $\mathcal{F}$ as the amplitude of the fit; see Supplementary Information), that is, no stored photons. Row (ii), resonator $R_{a}$ contains one photon $\left(\mathcal{F}=0.86 \mp 0.01\right.$ ), with the other two resonators in the vacuum state. In row (iii) the photon has been placed in $R_{b}$ $\left(\mathcal{F}=0.80 \mp 0.01\right.$ ), and in row (iv) the photon is in resonator $R_{c}(\mathcal{F}=0.69 \mp 0.01)$. In row ( $v$ ), we have taken the photon from $R_{c}$ and placed it back in $R_{b}$ $(\mathcal{F}=0.61 \mp 0.01)$, demonstrating the high degree of control and population coherence in the system. All data in the Letter are corrected for measurement errors (see Supplementary Information). 

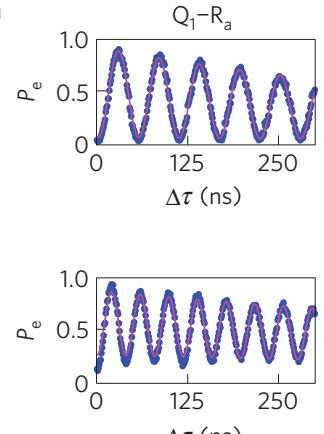

$\Delta \tau$ (ns)
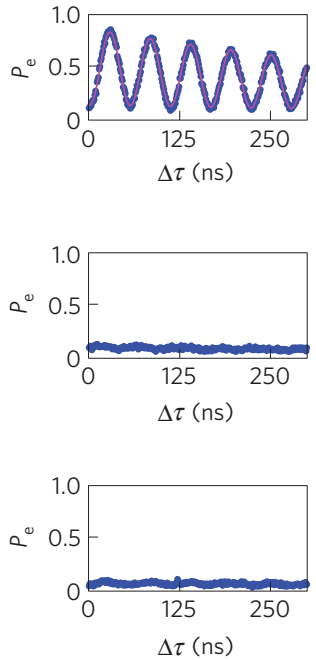
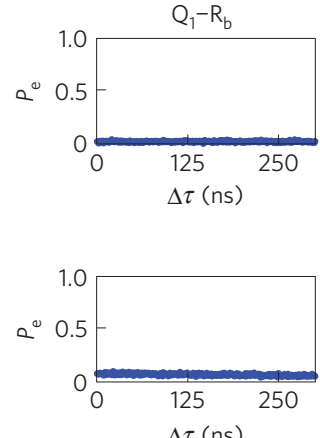

$\Delta \tau$ (ns)

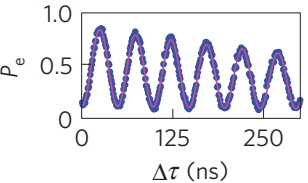

$\Delta \tau(\mathrm{ns})$
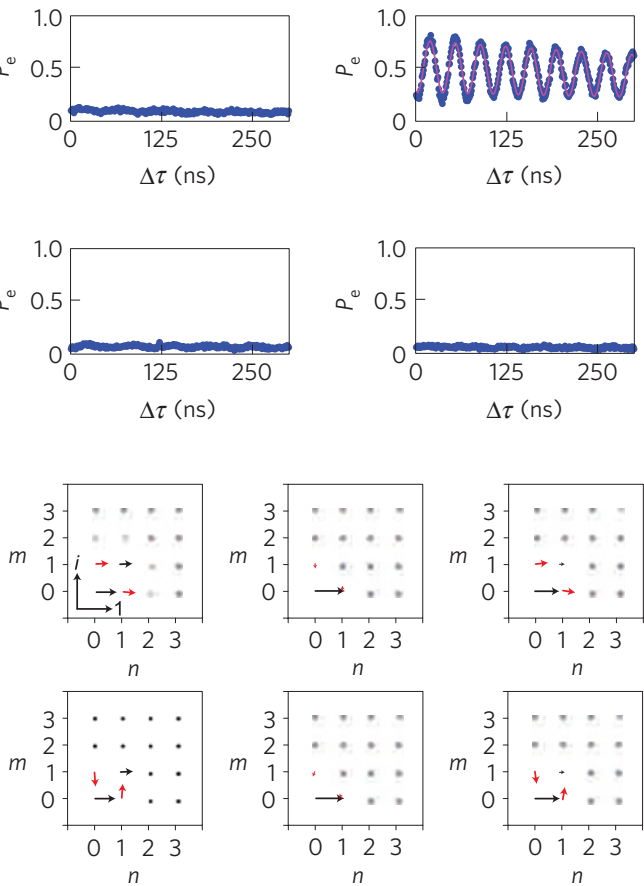
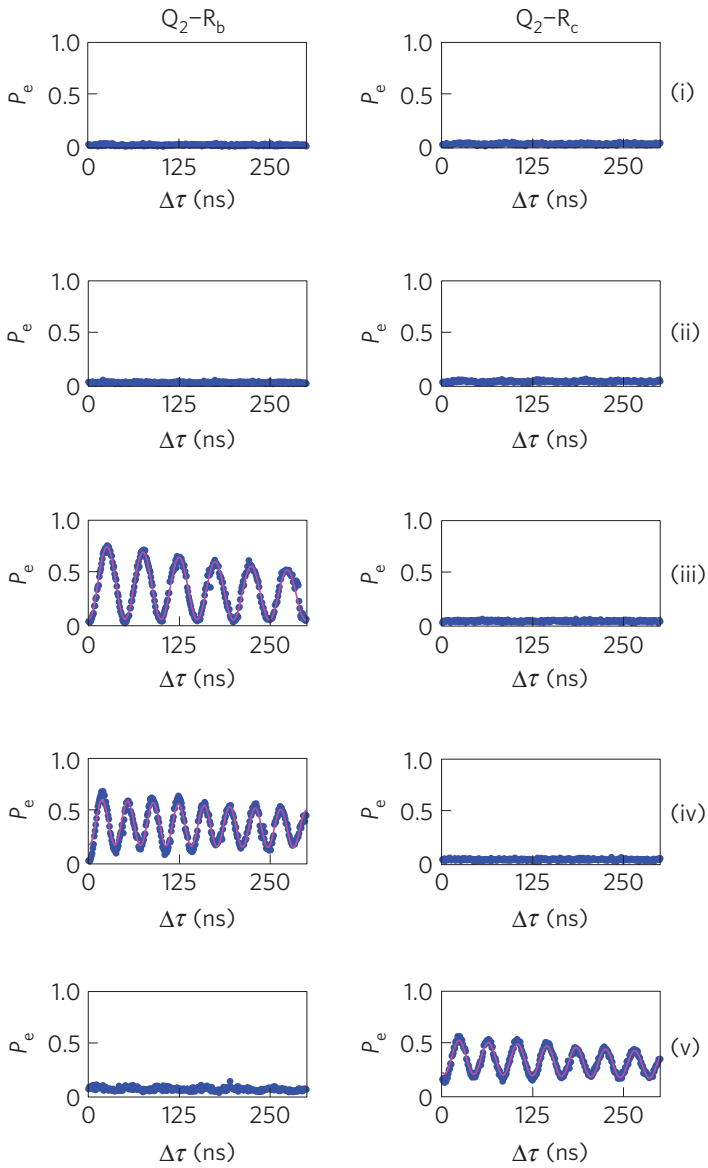

C
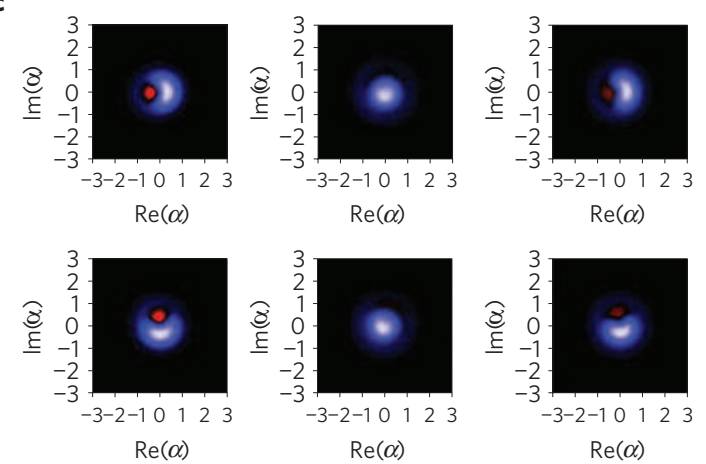

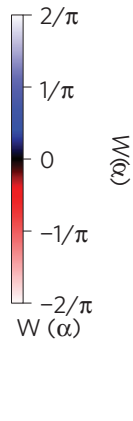

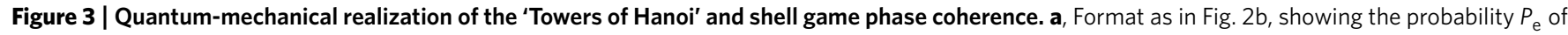

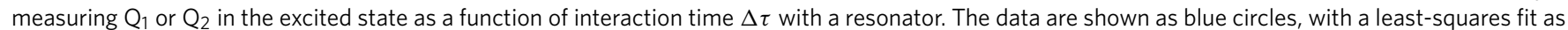
magenta solid lines. (i), A one-photon Fock state $|1\rangle$ in $R_{a}$ with both $R_{b}$ and $R_{c}$ in the vacuum state, and, (ii), a two-photon Fock state in $R_{a}$ (fidelity

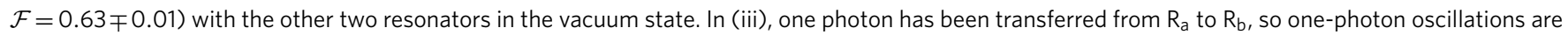
seen when $Q_{1}$ measures $R_{a}$ or $R_{b}$, and when $Q_{2}$ measures $R_{b}$. In (iv), the second photon has been transferred to $R_{b}(\mathcal{F}=0.51 \mp 0.01$ ), yielding the $\sqrt{2}$

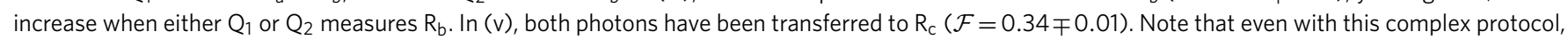
both $R_{a}$ and $R_{b}$ exhibit negligible oscillations $(\mathcal{F} \gtrsim 0.97)$. See Supplementary Information for further analysis. $\mathbf{b}$, From left to right: Density matrix $\hat{\rho}$ associated with resonator $R_{a}$ (resonator Hilbert space truncated to lowest four bosonic states) for $\left|\psi_{X}\right\rangle$ (top row) and $\left|\psi_{Y}\right\rangle$ (bottom row) in $R_{a}$, then

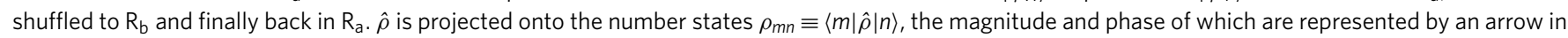
the complex plane, with scale embedded in the first panel. c, Wigner function $W(\alpha)$ associated with each $\hat{\rho}$ in $\mathbf{b}$, where $\alpha$ is the complex resonator amplitude in square root of photon number units (colour scale bar on the far right).

state $|1\rangle$, with the longer swapping time) always at the bottom of each post, and the smaller discs (Fock state $|2\rangle$, with shorter swapping time) on top.

Figure $3 b, c$ shows the density matrices and corresponding Wigner functions ${ }^{24}$ for the coherent transfer of the phase-sensitive state $\left|\psi_{X}\right\rangle=|0\rangle+|1\rangle$ (top panels) and $\left|\psi_{Y}\right\rangle=|0\rangle+\mathrm{e}^{i \pi / 2}|1\rangle$ (bottom panels) between $R_{a}$ and $R_{b}$. From left to right, each state is first prepared in $R_{a}$, shuffled to $R_{b}$, thus leaving $R_{a}$ in the vacuum state, and finally shuffled back to $\mathrm{R}_{\mathrm{a}}$. For a phase coherent transfer, the off-diagonal terms of the first and third density matrices on the top row (red arrows) should be orthogonal to those in the bottom row. We find a transfer orthogonality $\simeq 0.96$ and a total transfer fidelity $\simeq 0.92$ and $\simeq 0.88$ for $\left|\psi_{X}\right\rangle$ and $\left|\psi_{Y}\right\rangle$, respectively (see Supplementary Information). 


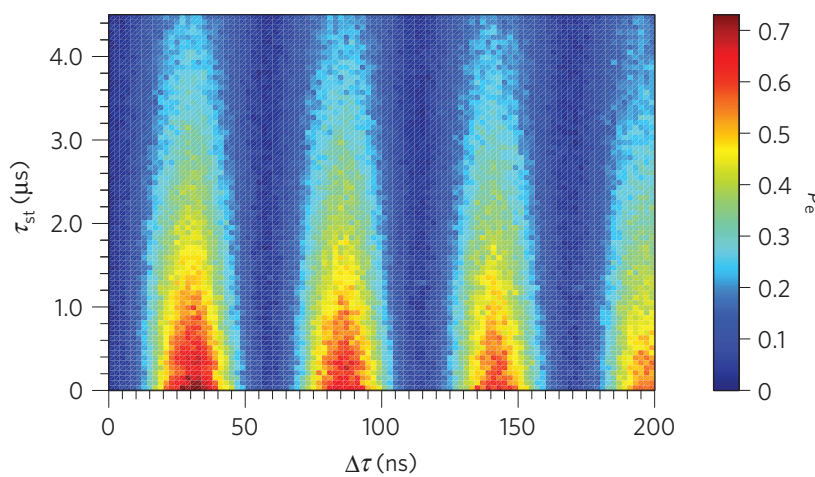

b

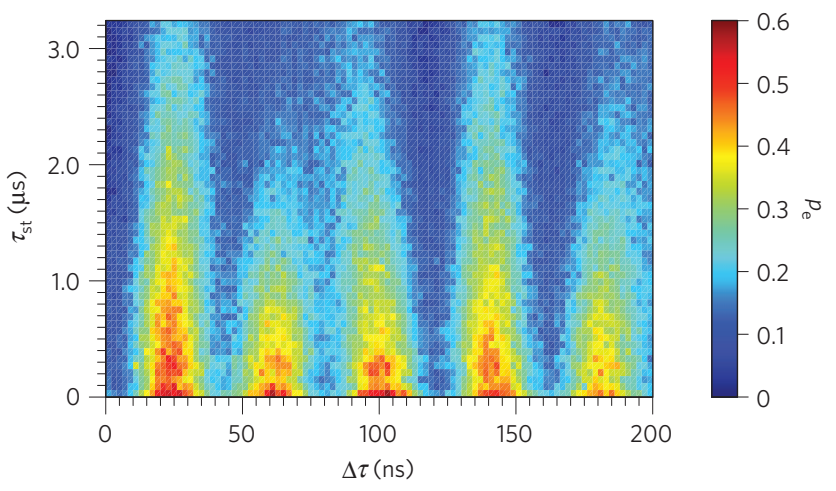

Figure 4 | Combined quantum state transfer and storage for one- and two-photon Fock states. a, Probability $P_{\mathrm{e}}$ to find $\mathrm{Q}_{2}$ in the excited state (colour bar scale, right side) versus measurement time $\Delta \tau$ (horizontal axis), and total storage time $\tau_{\text {st }}$ (vertical axis). A one-photon Fock state is created and stored in resonator $R_{a}$ for a time $\tau_{s t} / 3$, transferred to $R_{b}$ and stored for the same time, then transferred to $R_{c}$, stored for the same time and then measured. $\mathbf{b}$, The same as in $\mathbf{a}$, but for the generation and storage of a two-photon Fock state.

Another fundamental question for resonator-based quantum computing is whether quantum states can be stored in a resonator and later extracted and stored elsewhere. We demonstrate this functionality in Fig. 4, where a single photon is stored in each of the three resonators for a variable time $\tau_{\mathrm{st}} / 3$ before being transferred to the next resonator. Qubit measurements of the resonator containing the photon (for example resonator $R_{c}$ in Fig. 4a) exhibit clear oscillations for total storage times $\tau_{\text {st }}$ exceeding $3 \mu \mathrm{s}$. Figure $4 \mathrm{~b}$ is the same experiment repeated with a two-photon Fock state; clear oscillations are visible for total storage times $\tau_{\text {st }}$ exceeding $1.5 \mu$ s. These experiments demonstrate the realization of a programmable quantum information register.

We further show two-resonator vacuum Rabi swaps that can lead to quantum state entanglement between the two resonators $R_{a}$ and $\mathrm{R}_{\mathrm{b}}$. The protocol is diagrammed in Fig. 5a. A one-photon Fock state is first stored in $R_{a}$, placing the system in the state $\left|Q_{1} R_{a} R_{b}\right\rangle=|g 10\rangle$ (see Fig. $5 \mathrm{a}(\mathrm{I})-(\mathrm{III})$ ). The qubit is then used to carry out a partial transfer of the photon to $R_{b}$, by placing $Q_{1}$ in resonance with $R_{a}$ and varying the transfer time $\tau$ (see Fig. $5 \mathrm{a}(\mathrm{IV})$ ). This leaves the system in the entangled state $\alpha|\mathrm{g} 10\rangle+\beta|\mathrm{e} 00\rangle$, with amplitudes ${ }^{30}$ $\alpha=\cos \left(\pi g_{1 \mathrm{a}} \tau\right)$ and $\beta=-i \sin \left(\pi g_{1 \mathrm{a}} \tau\right)$. The qubit frequency $f_{1}$ is then tuned from $f_{\mathrm{a}}$ to $f_{\mathrm{b}}$, and left there for a time equal to the $\mathrm{Q}_{1}-\mathrm{R}_{\mathrm{b}}$ swap time, thus mapping the qubit state onto the resonator and resulting in the two-resonator entangled state

$$
\alpha|\mathrm{g} 10\rangle+\beta|\mathrm{g} 01\rangle=|\mathrm{g}\rangle \otimes(\alpha|10\rangle+\beta|01\rangle)
$$

We then use both qubits to simultaneously measure the two resonators, $\mathrm{Q}_{1}$ measuring $\mathrm{R}_{\mathrm{a}}$ for a measurement time $\Delta \tau_{1}$ and
$\mathrm{Q}_{2}$ measuring $\mathrm{R}_{\mathrm{b}}$ for a time $\Delta \tau_{2}$. Figure $5 \mathrm{~b}(\mathrm{c})$ shows the resulting oscillations in $P_{1 \mathrm{e}}\left(P_{2 \mathrm{e}}\right)$ for $\mathrm{Q}_{1}\left(\mathrm{Q}_{2}\right)$ (colour bar scale), as a function of the measurement time $\Delta \tau_{1,2}$ (horizontal axis) and $\mathrm{Q}_{1}-\mathrm{R}_{\mathrm{a}}$ transfer time $\tau$ (vertical axis). The data exhibit a single Rabi oscillation along the horizontal axis, which would repeat if the measurement time were increased, and also shows clear swaps as a function of the transfer time $\tau$, as expected from the functional dependence of $\alpha$ and $\beta$. If the measurement times $\Delta \tau_{1,2}$ are chosen to equal a full qubit-resonator swap time (dashed white lines in Fig. 5b,c), the system will be in the state $\left|\mathrm{Q}_{1} \mathrm{R}_{\mathrm{a}} \mathrm{R}_{\mathrm{b}} \mathrm{Q}_{2}\right\rangle=\alpha|\mathrm{e} 00 \mathrm{~g}\rangle+\beta|\mathrm{g} 00 \mathrm{e}\rangle$. An ideal measurement of $\mathrm{Q}_{1}$ and $\mathrm{Q}_{2}$ would then yield probabilities $P_{1 \mathrm{e}}=|\alpha|^{2}=\cos ^{2}\left(\pi g_{1 \mathrm{a}} \tau\right)$ and $P_{2 \mathrm{e}}=|\beta|^{2}=\sin ^{2}\left(\pi g_{1 \mathrm{a}} \tau\right)$ for $\mathrm{Q}_{1}$ and $\mathrm{Q}_{2}$, respectively, as a function of the transfer time $\tau$. In Fig. $5 \mathrm{~d}$ we show this functional dependence, with a clear $180^{\circ}$ phase difference between the two probabilities and the summed probability $P_{1 \mathrm{e}}+P_{2 \mathrm{e}}$ close to unity, as expected. The probabilities decrease with $\tau$, owing to the finite energy relaxation time of $\mathrm{Q}_{1}$ and $\mathrm{R}_{\mathrm{a}}$. The decay time of $\mathrm{R}_{\mathrm{b}}$ does not contribute noticeably (see Supplementary Information). Fitting yields an effective two-resonator decay time approximately equal to the harmonic mean of the energy relaxation times $T_{1}{ }^{\text {rel }} \simeq 340 \mathrm{~ns}$ and $T_{\mathrm{a}}^{\text {rel }} \simeq 3.9 \mu$ s of $\mathrm{Q}_{1}$ and $\mathrm{R}_{\mathrm{a}}$, respectively, $T_{\mathrm{ab}}{ }^{\text {rel }} \approx\left(1 / 2 T_{1}{ }^{\text {rel }}+1 / 2 T_{\mathrm{a}}{ }^{\mathrm{rel}}\right)^{-1} \simeq 626 \mathrm{~ns}$. We note that the phase qubit enables a photon transfer between $R_{a}$ and $R_{b}$ even though the two resonators are separated in frequency by $\simeq 12,000$ resonator linewidths.

This last experiment demonstrates a true quantum version of the 'shell game', where the 'pea' (the photon Fock state) is simultaneously hidden under two shells, and the contestant's selection of a shell constitutes a truly probabilistic measurement. More generally, we have experimentally demonstrated an architecture with three resonators and two qubits that exhibits excellent quantum control over single, double and superpositions of microwave photon Fock states. From a fundamental perspective, these results demonstrate the potential of multi-resonator circuit QED (refs 27,28), both for scientific study and for quantum information.

\section{Methods}

Sample fabrication. The resonators are made from a 150-nm-thick rhenium film grown in a molecular-beam-epitaxy system on a polished sapphire substrate. All the other wiring layers are made from sputtered aluminium with $\mathrm{Al} / \mathrm{AlO}_{x} / \mathrm{Al}$ Josephson tunnel junctions. All of the microstructures on the different layers are patterned by means of optical lithography and etched by means of inductively coupled plasma etching. Amorphous silicon is used as a dielectric insulator for the qubit shunting capacitors and crossovers. Our sample fabrication clearly shows the flexibility offered by multi-layer processing.

The complete device is wire bonded by aluminium wire-bonds to an aluminium sample holder, which is bolted to the mixing chamber of a dilution refrigerator operating at $\simeq 25 \mathrm{mK}$. A detailed description of the fabrication techniques, electronics and qubit calibration procedures can be found elsewhere ${ }^{23}$.

Three-resonator circuit QED Hamiltonian. The Hamiltonian $\widehat{H}$ for the circuit of Fig. $1 \mathrm{~b}$ can be written as the sum of the Hamiltonians of each unit cell, $\widehat{H}_{1}$ and $\widehat{H}_{2}$. Neglecting the driving and dissipative terms for simplicity, the Hamiltonian of the first circuit unit cell can be expressed in the interaction picture with respect to $Q_{1}$ and $\mathrm{R}_{\mathrm{a}}$ and $\mathrm{R}_{\mathrm{b}}$ as the combination of two Jaynes-Cummings interactions:

$$
\begin{aligned}
\widehat{H}_{1}= & h \frac{g_{1 \mathrm{a}}}{2}\left(\hat{\sigma}_{1}^{+} \hat{a} \mathrm{e}^{+i 2 \pi \Delta_{1 \mathrm{a}} t}+\hat{\sigma}_{1}^{-} \hat{a}^{\dagger} \mathrm{e}^{-i 2 \pi \Delta_{1 \mathrm{a}} t}\right) \\
& +h \frac{g_{1 \mathrm{~b}}}{2}\left(\hat{\sigma}_{1}^{+} \hat{b} \mathrm{e}^{+i 2 \pi \Delta_{1 \mathrm{~b}} t}+\hat{\sigma}_{1}^{-} \hat{b}^{\dagger} \mathrm{e}^{-i 2 \pi \Delta_{\mathrm{bb}} t}\right)
\end{aligned}
$$

where $\hat{\sigma}_{1}^{ \pm}$are the rising and lowering operators for $\mathrm{Q}_{1}, \hat{a}, \hat{a}^{\dagger}, \hat{b}$ and $\hat{b}^{\dagger}$ are the bosonic annihilation and creation operators for $\mathrm{R}_{\mathrm{a}}$ and $\mathrm{R}_{\mathrm{b}}$, respectively, and $\Delta_{1 \mathrm{a}} \equiv f_{1}-f_{\mathrm{a}}$ and $\Delta_{1 \mathrm{~b}} \equiv f_{1}-f_{\mathrm{b}}$ are the qubit-resonator detunings. The Hamiltonian $\hat{H}_{2}$ for the second circuit unit cell has an analogous expression.

To effectively switch off a particular qubit-resonator $\mathrm{Q}_{p}-\mathrm{R}_{q}$ interaction, the condition $\Delta_{p q} \gg g_{p q}$ must be fulfilled. This is the case when $\mathrm{Q}_{1}\left(\mathrm{Q}_{2}\right)$ is at the idle point. On the contrary, when $\Delta_{p q} \rightarrow 0$, a resonant Jaynes-Cummings interaction takes place enabling state preparation and transfer in and between the resonators as well as photon detection. 


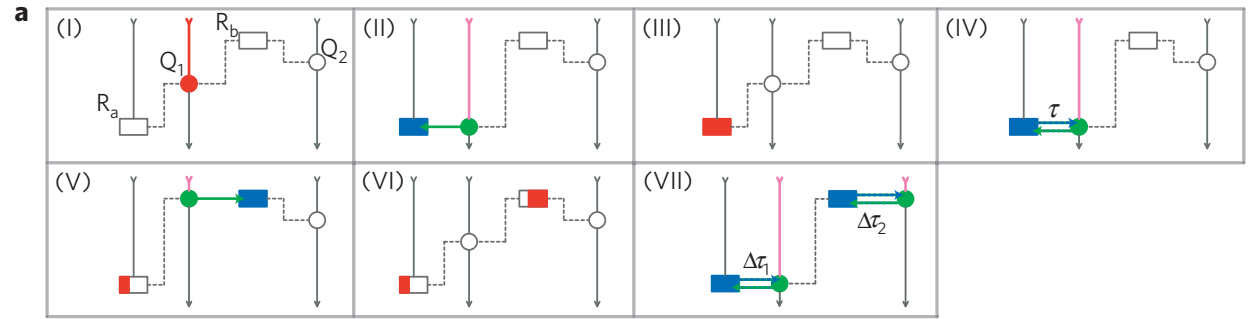

b

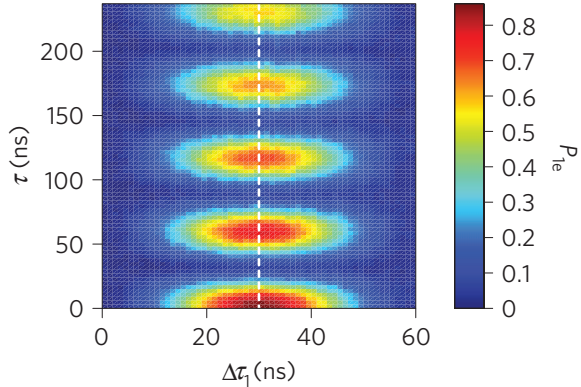

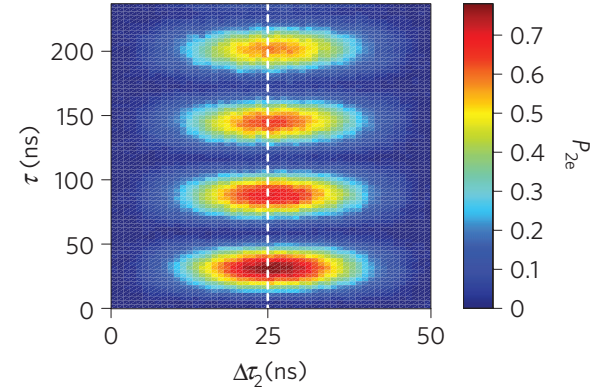

d

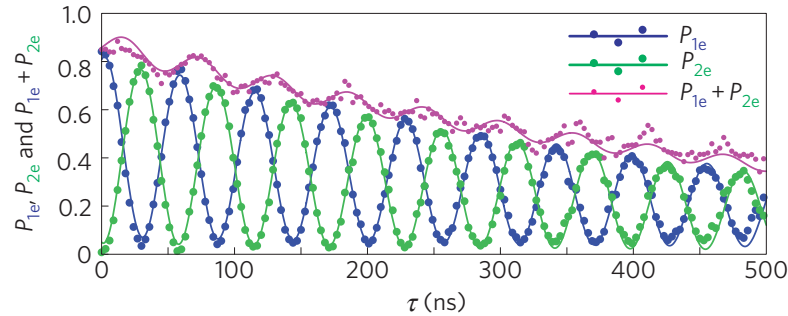

Figure 5 | Two-resonator Rabi swaps. a, Block diagram of the preparation and measurement protocol. (I), $Q_{1}$ is excited by a $\pi$-pulse and, (II), brought into resonance with $R_{a}$ for a Rabi $\pi$-swap, at the end of which, (III), $R_{a}$ is populated by a one-photon Fock state. In (IV), $Q_{1}$ is brought into resonance with $R_{a}$ for a variable transfer time $\tau$ at the end of which, $(V), R_{a}$ is left partially populated, and, $(V)$ and (VI), the remaining energy is fully transferred to $R_{b}$ by means of a Rabi $\pi$-swap with $Q_{1}$. The probabilities for having the photon in $R_{a}$ or $R_{b}$ can be varied continuously by changing the transfer time $\tau$ (see main text). These probabilities are simultaneously measured for $R_{a}$ with $Q_{1}$ and for $R_{b}$ with $Q_{2}(V I I)$. b, Qubit probability $P_{1 e}$ (colour scale bar) as a function of the $\mathrm{Q}_{1}-\mathrm{R}_{\mathrm{a}}$ measurement time $\Delta \tau_{1}$ (horizontal axis) and the transfer time $\tau$ (vertical axis). $\mathbf{c}$, The same as in $\mathbf{b}$, but for $\mathrm{Q}_{2}$ 's probability $P_{2 \mathrm{e}}$ as a function of the $\mathrm{Q}_{2}-\mathrm{R}_{\mathrm{b}}$ measurement time $\Delta \tau_{2}$. Measurement times are sufficient to show one complete Rabi oscillation between the measurement qubit and resonator, with a Rabi swap occurring at the centre of each horizontal axis (dashed white line), with multiple swaps shown as a function of the $Q_{1}-R_{a}$ transfer time $\tau$ (vertical direction). Cuts through the probabilities along the dashed white lines (full Rabi swaps) are shown in d, where the expected co-sinusoidal oscillations are observed in $\mathrm{Q}_{1}$ 's probability $P_{1 \mathrm{e}}$ (dark blue circles) $\left(\mathrm{Q}_{2}\right.$ 's probability $P_{2 \mathrm{e}}$; light green circles), with the summed probability $P=P_{1 \mathrm{e}}+P_{2 \mathrm{e}}$ (magenta circles) showing the expected slow decay (see main text). The solid lines are fits to data.

\section{Received 5 August 2010; accepted 12 November 2010;} published online 30 January 2011

\section{References}

1. Mabuchi, H. \& Doherty, A. C. Cavity quantum electrodynamics: Coherence in context. Science 298, 1372-1377 (2002).

2. Haroche, S. \& Raimond, J-M. Exploring the Quantum (Oxford Univ. Press, 2006).

3. Walther, H., Varcoe, B. T. H., Englert, B-G. \& Becker, T. Cavity quantum electrodynamics. Rep. Prog. Phys. 69, 1325-1382 (2006).

4. Rauschenbeutel, A. et al. Controlled entanglement of two field modes in a cavity quantum electrodynamics experiment. Phys. Rev. A 64, 050301(R) (2001).

5. Gleyzes, S. et al. Quantum jumps of light recording the birth and death of a photon in a cavity. Nature 446, 297-300 (2007)

6. Deléglise, S. et al. Reconstruction of non-classical cavity field states with snapshots of their decoherence. Nature 455, 510-514 (2008).

7. Hijlkema, M. et al. A single-photon server with just one atom. Nature Phys. 3, 253-255 (2007).

8. Wilk, T., Webster, S. C., Kuhn, A. \& Rempe, G. Single-atom single-photon quantum interface. Science 317, 488-490 (2007).

9. Dayan, B. et al. A photon turnstile dynamically regulated by one atom. Science 319, 1062-1065 (2008)

10. Papp, S. B. et al. Characterization of multipartite entanglement for one photon shared among four optical modes. Science 324, 764-768 (2009).

11. Wallraff, A. et al. Strong coupling of a single photon to a superconducting qubit using circuit quantum electrodynamics. Nature 431, 162-167 (2004).
12. Chiorescu, I. et al. Coherent dynamics of a flux qubit coupled to a harmonic oscillator. Nature 431, 159-162 (2004).

13. Johansson, J. et al. Vacuum Rabi oscillations in a macroscopic superconducting qubit LC oscillator system. Phys. Rev. Lett. 96, 127006 (2006).

14. Schoelkopf, R. J. \& Girvin, S. M. Wiring up quantum systems. Nature 451, 664-669 (2008)

15. Makhlin, Yu., Schön, G. \& Shnirman, A. Quantum-state engineering with Josephson-junction devices. Rev. Mod. Phys. 73, 357-400 (2001).

16. Wendin, G. \& Shumeiko, V. S. in Handbook of Theoretical and Computational Nanotechnology Vol. 3 (eds Rieth, M. \& Schommers, W.) 223-309 (American Scientific, 2006).

17. You, J. Q. \& Nori, F. Superconducting circuits and quantum information. Phys. Today 58, 42-47 (November, 2005).

18. Clarke, J. \& Wilhelm, F. K. Superconducting quantum bits. Nature 453, 1031-1042 (2008).

19. Houck, A. A. et al. Generating single microwave photons in a circuit. Nature 449, 328-331 (2007).

20. Sillanpää, M. A., Park, J. I. \& Simmonds, R. W. Coherent quantum state storage and transfer between two phase qubits via a resonant cavity. Nature 449, 438-442 (2007).

21. Hofheinz, M. et al. Generation of Fock states in a superconducting quantum circuit. Nature 454, 310-314 (2008).

22. Deppe, F. et al. Two-photon probe of the Jaynes-Cummings model and controlled symmetry breaking in circuit QED. Nature Phys. 4, 686-691 (2008).

23. Hofheinz, M. et al. Synthesizing arbitrary quantum states in a superconducting resonator. Nature 459, 546-549 (2009).

24. Wang, H. et al. Decoherence dynamics of complex photon states in a superconducting circuit. Phys. Rev. Lett. 103, 200404 (2009). 
25. Leek, P. J. et al. Cavity quantum electrodynamics with separate photon storage and qubit readout modes. Phys. Rev. Lett. 104, 100504 (2010).

26. Johnson, B. R. et al. Quantum non-demolition detection of single microwave photons in a circuit. Nature Phys. 6, 663-667 (2010).

27. Mariantoni, M. et al. Two-resonator circuit quantum electrodynamics: A superconducting quantum switch. Phys. Rev. B 78, 104508 (2008).

28. Helmer, F. et al. Cavity grid for scalable quantum computation with superconducting circuits. Europhys. Lett. 85, 50007 (2009).

29. Sun, C. P., Wei, L. F., Liu, Y-X. \& Nori, F. Quantum transducers: Integrating transmission lines and nanomechanical resonators via charge qubits. Phys. Rev. A 73, 022318 (2006).

30. Walls, D. F. \& Milburn, G. J. Quantum Optics 2nd edn (Springer, 2008).

\section{Acknowledgements}

This work was supported by IARPA under ARO award W911NF-08-1-0336 and under ARO award W911NF-09-1-0375. M.M. acknowledges support from an Elings Prize
Postdoctoral Fellowship. Devices were made at the UC Santa Barbara Nanofabrication Facility, a part of the NSF-funded National Nanotechnology Infrastructure Network.

\section{Author contributions}

M.M. carried out the experiments with the help of H.W. M.M. analysed the data and carried out the numerical simulations. M.M. and H.W. fabricated the sample. M.N. provided software infrastructure. J.M.M. and E.L. designed the custom electronics. E.L. took the sample picture. All authors contributed to the fabrication process, qubit design or experimental set-up, and discussed the data analysis. M.M., J.M.M. and A.N.C. conceived the experiment and co-wrote the paper.

\section{Additional information}

The authors declare no competing financial interests. Supplementary information accompanies this paper on www.nature.com/naturephysics. Reprints and permissions information is available online at http://npg.nature.com/reprintsandpermissions. Correspondence and requests for materials should be addressed to M.M. or A.N.C. 